\title{
La subjetivación de las relaciones económicas. Reflexiones para una tipología de mercados informales de arrendamiento residencial
}

\author{
Hernando Sáenz Acosta \\ Programa de Estudantes-Convênio de Pós-Graduação (PEC-PG), Brasil \\ Email: hernandosaenz@gmail.com
}

Resumen $^{1}$ : El objetivo es presentar unas reflexiones teóricas sobre la informalidad en los mercados de arrendamiento residencial que tienen lugar en los barrios de origen informal de Bogotá. Se considera como fuente de informalidad la subjetivación de las relaciones económicas y contractuales entre los agentes que participan en este mercado, cuyo fin es garantizar la reproducción ampliada de la vida. A partir de esa hipótesis se analizan las interpretaciones proporcionadas por las teorías de la marginalidad, de la dependencia y de la informalidad sobre el barrio de origen informal como espacio de subjetivación de relaciones sociales y económicas. Teniendo en cuenta esa revisión se propone una tipología de mercados informales por medio de la cual sea posible analizar con mayor detalle estos procesos de acceso a la vivienda por parte de las familias de menores ingresos en esta ciudad.

Palabras clave: vivienda, mercado de arrendamiento, informalidad, reciprocidad, asentamientos de origen informal.

\section{The subjectivity of economics relations. Reflections for a tipology of informal markets residential lease}

\begin{abstract}
The aim is to show some theoretical reflections about the informal residential lease market, especially in neighborhoods which origins are informal in the city of Bogotá. The economics and social relations are personalized by the agents like strategy for to secure the expanded reproduction of human life. This hypothesis is the starting point to analyze different interpretations about theories like marginality, dependency and informality and the considerations of the popular neighborhoods like space of social and economic subjective relations. This analysis allows build an informal markets typology and, understand the process to access to dwelling by families with low income in this city.

Keywords: housing, lease market, informality, reciprocity, popular neighborhood.
\end{abstract}




\section{A subjetivación das relações economicas. Reflexões para uma tipologia de mercados informais de aluguel residencial}

Resumo: O objetivo deste artigo consiste em apresentar algumas reflexões teóricas sobre a informalidade nos mercados de aluguel que tem lugar nos bairros de origem informal de Bogotá. Se considera como fonte de informalidade a subjetivação das relações econômicas e contratuais entre os agentes que participam neste mercado, cuja finalidade é garantir a reprodução ampliada da vida. A partir dessa hipótese analisam-se as interpretações proporcionadas pelas teorias da marginalidade, da dependência e da informalidade sobre o bairro de origem informal como espaço de subjetivação de relações sociais e econômicas. Tendo em conta essa revisão propõese uma tipologia de mercados informais por meio da qual seja possível analisar com maior detalhe estes processos de acesso à vivenda por parte das famílias de menor renda nesta cidade.

Palavras-chave: vivenda, mercado de aluguel, informalidade, reciprocidade, assentamentos de origem informal

$* * *$

\section{Introducción}

Una de las principales formas de acceso a la vivienda en Bogotá corresponde al arrendamiento. Según los datos censales dicha población pasó de representar un 39,9\% en 1985 para situarse en un 41,6\% en 1993 y 43\% en 2005 (Torres, 2012). En 2011 la proporción de hogares que pagaban arriendo se encontraba en un 41,4\% aglutinando unas 904.893 familias de las cuales un 14,9\% tenía un ingreso inferior a un salario mínimo mensual y un 28,9\% tenían ingresos situados entre uno y dos salarios mínimos. Así pues, sumados alcanzaban a representar el 43,8\% (CENAC, 2012).

En cuanto a la localización de estos hogares en la ciudad, se observa que la mayoría vive en barrios de estratos uno, dos y tres ${ }^{2}$. En el primer caso se trata de $8,2 \%$, en el segundo de $44,7 \%$ y en el tercero de $36,7 \%$ del total de familias arrendatarias (CENAC, 2012). Si tenemos en cuenta que por lo general buena parte de los barrios que surgen al margen de la normatividad urbanística pertenecen a los dos primeros estratos, se puede afirmar que un $52,9 \%$ de las familias que viven en arriendo en Bogotá residiría en barrios de origen informal.

La magnitud del arriendo residencial en los barrios de origen informal ya ha sido destacada en algunas investigaciones como una de las principales formas de acceso a la vivienda por parte de las familias con menores ingresos de Bogotá (Parias, 2010). Desde el punto de vista de los arrendadores, constituye una fuente complementaria de ingresos y para los hogares arrendatarios una posibilidad de acceder a la vivienda cuando no es posible hacerlo en propiedad sea por la vía del mercado formal o informal. Así pues se trata de un mercado donde las partes que intervienen conser- 
van relativas semejanzas en términos socioeconómicos (CENAC, 2007; Parias \& Abramo, 2009).

Una segunda característica relacionada con el mercado de arrendamiento en estos barrios se refiere a la existencia de redes de relaciones que son territorializadas. Estas redes (parentesco, amistad, vecindad) constituyen un recurso importante para el acceso a la vivienda, así como a otros bienes y servicios indispensables para la reproducción socioeconómica de la familia (Parias 2010). No obstante, dicha presencia de redes es asociada también con una informalidad contractual, ya que al prevalecer intercambios entre amigos, parientes, se tiende a establecer contratos de palabra u orales. La existencia de este tipo de contratos se considera negativa porque no especifican claramente los derechos de propiedad, fuente de conflictos entre inquilinos y arrendadores que pueden ser costosos de solucionar, especialmente cuando se aplican mecanismos violentos (CENAC, 2007; ONU, 2012) ${ }^{3}$.

La ausencia de una regulación estatal, no obstante, no parece ser un problema para el desarrollo de este mercado pues se ha comprobado la existencia de sistemas de regulación creados y desarrollados por las propias comunidades que apelan a las costumbres, las normas, la tradición para garantizar la cooperación y coordinación entre los agentes, así como para la resolución (sea por vías pacíficas o violentas) de los conflictos con o sin la presencia de un tercer actor (sea el Estado o algún otro tipo de autoridad comunitaria). La principal forma de regulación consiste en la reciprocidad, la cual actúa como un marco institucional de los contratos que se realizan en este mercado (Abramo, 2009).

La hipótesis que constituye el punto de partida de este trabajo deriva de observar la importancia que tienen las redes de relaciones en el mercado de arrendamiento presente en los asentamientos de origen informal. Ellas permiten que los agentes - en virtud de la existencia de relaciones de parentesco, amistad, etc.,-- puedan personalizar o subjetivar una relación de mercado que es anónima e individual y por medio de esa subjetivación acceder a una vivienda en arriendo, incidir en la determinación del precio y/ o aplicar mecanismos de resolución de conflictos que no necesariamente sean tramitados desde el Estado.

Así pues, el objetivo de este artículo consiste en tomar como referente la hipótesis de personalización o subjetivación de las relaciones económicas y contractuales para la construcción de una tipología de mercados informales de arrendamiento ${ }^{4}$. En ese sentido se aborda en la primera parte del texto una referencia sobre los primeros trabajos destinados a la comprensión de la urbanización y el predominio de relaciones sociales y económicas objetivas en las ciudades modernas, para luego dar paso a la interpretación que se presentó al respecto sobre la urbanización latinoamericana. En la tercera y última parte se presenta la tipología de mercados resultante y una serie de conclusiones relacionadas con la construcción de una hipótesis de trabajo que considera la subjetivación de la relación económica 
como estrategia desarrollada por las familias con el ánimo de garantizar la reproducción ampliada de la vida humana.

\section{La ciudad moderna: escenario de relaciones sociales objetivadas}

Una de las consecuencias del proceso urbanizador que comenzó en el siglo XIX ha sido la consolidación de una economía monetaria que promueve la objetivación de las relaciones sociales. Esa objetivación descrita por autores como Simmel se caracterizaba por el predominio de individuos que se comportaban de forma racional, evaluando su relación con las cosas y demás personas según criterios pecuniarios. La metrópolis se identificaba como el lugar de la individualidad y de la existencia personal porque la característica distintiva de la vida moderna era la dominación de un espíritu objetivo sobre otro subjetivo. El desarrollo de la libertad individual se favorecía por la creciente especialización y división del trabajo siendo uno de sus principales obstáculos la persistencia de las relaciones primarias, especialmente de aquellas basadas en el parentesco (Simmel, 1903/1988).

En lo que concierne al caso de urbanización de Estados Unidos, la Escuela de Sociología de Chicago realizó un análisis de ese proceso de objetivación de las relaciones sociales y las dinámicas migratorias para postular que ella tenía efectos positivos en la medida en que se promovía un mayor desarrollo intelectual de los individuos, razas, y comunidades, situación que contrastaba con la inmovilidad y la tendencia a sostener vínculos íntimos con los lugares y personas (Park, 1936/1999).

La objetivación de las relaciones sociales implica también la substitución de un control social basado en las costumbres y hábitos por otros donde imperan leyes positivas. En la ciudad moderna el paso de uno a otro control social se caracteriza por 1) la substitución de la costumbre por la ley positiva y la extensión del control municipal a actividades que eran antes de iniciativa individual; 2) la entrega de funciones administrativas a los jueces en los tribunales municipales y locales y 3 ) mudanzas y cambios en las costumbres de los distintos grupos urbanos aislados o segregados. Este proceso de objetivación se observaría especialmente entre los migrantes que llegarían a vivir en las ciudades estadounidenses, símbolos de una organización racional (civilización) basada en intereses racionales y preferencias temperamentales (Park, 1998; Stonequist, 1935).

La objetivación de las relaciones sociales aparece finalmente como símbolo del modo de vida urbana en la cual las relaciones interpersonales son utilitarias, promovidas especialmente por la mayor especialización y división del trabajo. Sin embargo y en virtud de la mayor movilidad, se van a generar fidelidades intermitentes a diferentes grupos, que en el peor de los casos lleva a una fragmentación de la personalidad (Wirth, 1973). Ya el mismo Simmel afirmaba que en la medida en que avanzaba una 
despersonalización de las relaciones sociales se presentaba una atrofia de la cultura individual y una hipertrofia de la cultura objetiva, mientras que Park afirmaba que la excesiva movilidad colocaba a los individuos en situación de vulnerabilidad frente a procesos de manipulación de masas por parte de instituciones como la ciudad-mercado y la bolsa de valores (Simmel, 1988; Park, 1998).

Es a partir de estas consideraciones iniciales que se considera pertinente analizar la interpretación que se hizo del proceso urbanizador latinoamericano, así como de la objetivación de las relaciones sociales, pues de ella vino posteriormente un debate que se considera importante para analizar el papel de las redes de relaciones en el mercado de arrendamiento que tiene lugar en los barrios de origen informal.

\section{La ciudad latinoamericana: el debate acerca de la objetivación parcial de las relaciones sociales}

Una de las primeras interpretaciones del proceso urbanizador latinoamericano se sirvió de los aportes de la Escuela de Sociología de Chicago. No obstante a diferencia del caso estadounidense, se observaba la presencia de sectores arcaicos y modernos, lo que hizo que autores como Gino Germani calificaran el proceso latinoamericano como de modernización parcial, ya que a pesar de la consolidación de las clases medias (lo que era interpretado como símbolo de modernización) se había constituido otro sector integrado en su mayoría por una población de origen campesino que había migrado a las ciudades y que si bien no poseía el mismo nivel de vida de la clase media sí experimentaba mejoras en relación con lo observado en las zonas rurales (Germani, 1969).

Esa presencia de un sector arcaico fue denunciada especialmente en las ciudades latinoamericanas donde comenzaron a aparecer asentamientos producidos al margen de las normatividades urbanísticas. El Centro de investigación llamado Desarrollo Social para América Latina DESAL adaptó en los años sesenta el concepto de hombre marginal propuesto en la Escuela de Sociología de Chicago para caracterizar al tipo de poblador que habitaba en estos asentamientos al cual presentaban como antítesis del hombre moderno (Cortes, 2002; Bassols, 1990).

Se observaba un desequilibrio entre la tasa de urbanización y la de crecimiento industrial, fenómeno conocido como hiperurbanización. Así, lejos de una integración por la vía del mercado laboral, los migrantes tenían que dedicarse a actividades de baja productividad o engrosar la proporción de población sin empleo estable o en abierta situación de desempleo. La comparación con los casos europeo y estadounidense llevó a plantear el caso latinoamericano como una aberración o patología y definir la pobreza urbana como la no integración de los migrantes al sector moderno de la economía (Schteingart, 2000).

Tomando como referente la Escuela Francesa de Sociología Urbana y las teorías de la dependencia, surge una segunda lectura del proceso 
urbanizador en la región que parte de identificar la ciudad moderna como ciudad capitalista en la cual predomina la consecución de lucros y del Estado como el principal promotor de esa finalidad. La ciudad como forma urbana es vista como red de relaciones de producción, las cuales son el fundamento de las relaciones sociales. Las relaciones sociales dominantes condicionan el mercado de tierras urbanas siendo la morfología urbana resultado de los requerimientos de la producción capitalista (CEO, 2002).

La sociedad urbana identificada con la sociedad resultante de la industrialización posee un sistema de valores que se expande más allá de la ciudad y que se define específicamente por una sociedad donde el capital tiende a destruir las formas de consumo de espacio que no se ajustan a las relaciones mercantiles. La ciudad es un instrumento de reproducción del capital, una mercancía-producto y constituye la herramienta para organizar la producción, controlar la vida cotidiana de los productores y consumidores y ajustar el consumo de los productos (Valencia, 2005; Lefebvre, 1972, 1973; CEO, 2002).

Autores como Manuel Castells destacan el papel de la ciudad como unidad territorial de reproducción de la fuerza de trabajo. Existen los llamados medios de consumo colectivo, objetivamente socializados y que por motivos históricos específicos depende de la intervención del Estado para su producción, comercialización y gestión. Otros como Cristian Topalov se refieren a la urbanización capitalista y el surgimiento de monopolios de la renta del suelo cuyo efecto es la generación de una segregación socioespacial. Para ambos autores la participación del Estado en el espacio urbano es importante ya que transfiere los costos de la fuerza de trabajo al conjunto de la sociedad (Valencia, 2005; Castells, 1974; Topalov, 1979).

Desde este punto de vista se construyó una interpretación del proceso urbanizador latinoamericano que fue complementado por la teoría de la dependencia. Dicha urbanización estaba en función de los procesos de acumulación de capital a nivel internacional, cuyo centro estaba geográficamente localizado en Europa y los Estados Unidos. En América Latina el proceso de acumulación dependiente estará asociado a la generación de una proletarización parcial de la población, mientras que permanecerán grandes segmentos de la población excedente, la cual lejos de ser vistas como inadaptados culturales (lectura de la teoría de la marginalidad) corresponde a una población para la cual el Estado y el Capital no generan las condiciones básicas para la reproducción de su fuerza de trabajo (Jaramillo, 1993).

Uno de los principales aspectos necesarios para la reproducción de la fuerza de trabajo se refiere a la vivienda. Desde esta visión se explica entonces la permanencia de formas de producción y circulación del espacio construido no capitalistas, la tolerancia e incluso promoción desde el Estado. El acceso al suelo y la vivienda a través de estas formas no capitalistas están presentes en la mayoría de las ciudades latinoamericanas. La vivienda autoconstruida es identificada como forma no capitalista, caracterizán- 
dose por la construcción progresiva del lote, mientras que en términos de equipamientos colectivos y específicamente de servicios públicos domiciliarios se recurre a las redes de clientelismo o a la movilización social (Jaramillo, 1980, 1999).

El mercado de arrendamiento surge en estos barrios porque constituye para los propietarios de las casas autoconstruidas, la opción de obtener ingresos complementarios a las que obtienen de sus actividades laborales y para los arrendatarios la opción de acceder a la vivienda cuando no logran acceder en propiedad (formal o informalmente). La utilización de las redes de relaciones aparece aquí lejos de su dimensión cultural como en el caso de la teoría de la marginalidad para interpretarse como un recurso funcional por el cual se intenta paliar las deficiencias estatales y mercantiles (Jaramillo, 1999).

La tercera lectura sobre la urbanización latinoamericana surge en los años ochenta y se apoya en el concepto de informalidad, el cual se asocia con la crisis del modelo fordista de producción y sus efectos en el mercado del trabajo. Esta teoría analizó las diferentes prácticas y estrategias desarrolladas por las poblaciones que habitaban en los asentamientos autoproducidos en las ciudades de los países llamados subdesarrollados. La tesis principal de esta corriente es que la pobreza deriva de la imposibilidad de transformar los activos (principalmente vivienda) en capital ya que no estarían claramente definidos los títulos de propiedad. Dicha ausencia de título es culpa de un Estado que puede revertir la situación en la medida en que legalice los activos, iniciando así una senda de crecimiento económico desde estos barrios de origen informal (De Soto, 2001).

Según De Soto la imposibilidad de formalizar los activos explica la existencia de las redes de relaciones y el predominio de grupos y relaciones primarias en las principales actividades económicas. Los habitantes de estos asentamientos son vistos como pequeños emprendedores que desarrollan acuerdos y compromisos informales con el fin de proteger y movilizar sus activos. El resultado es un mundo sub-capitalizado por esa condición de extra-legalidad. Los acuerdos son resultado de una combinación de reglas tomadas del sistema legal oficial y del otro lado improvisaciones, costumbres traídos ad hoc de sus lugares de origen o producidos localmente. Existe así un contrato social apoyado por la comunidad como un todo y reforzado por autoridades que son escogidas por ella misma. Esta informalización tiene entonces la desventaja de restringir los circuitos de comercio a círculos locales donde los intercambios se realizan entre amigos y/o familiares (De Soto, 2001).

La intervención del Estado se centra así en el reconocimiento de los acuerdos extra-legales y su inclusión en la legalidad con lo cual se haría posible la consolidación de un mercado expandido, un mercado de economía moderno. El derecho aparece en esta lectura como una convención fusionando costumbres, hábitos y normas legales alrededor de una institucionalidad propia que le permite a los sectores desfavorecidos su 
integración al sector formal. En este caso, los sectores que viven en estos asentamientos actúan siguiendo una lógica de costo-beneficio por la cual la informalidad termina siendo la opción más eficiente en virtud del costo que implica integrarse a los sistemas legales de propiedad (De Soto, 2001).

\section{A manera de conclusión preliminar: la subjetivación de la relación económica en el mercado informal de arrendamiento residencial.}

A partir de la revisión de las tres principales lecturas de la urbanización latinoamericana se puede afirmar que la objetivación de las relaciones sociales y económicas no se alcanza en el caso de la urbanización latinoamericana y en términos más específicos de que el mercado informal de arrendamiento que tiene lugar en estos barrios constituye un ejemplo de situaciones donde lo social continua determinando lo económico. Dicho encuadramiento de lo económico en lo social fue mencionado por autores como Karl Polanyi (2013a, 2013b) para denunciar la falacia de mercado. Esta falacia consiste en pensar que lo económico se refiere exclusivamente a la lógica de la acción racional y la elección entre distintos usos de los medios que es inducida por la insuficiencia de dichos medios, la escasez. Dicha concepción formal de lo económico se contrapone a una definición substantiva en la cual se hace hincapié en la constatación de que la subsistencia del hombre depende de la naturaleza y de sus semejantes, el cual puede acontecer en situaciones de escasez pero no ser la única.Así la definición substantiva engloba a la formal y es utilizada por él para construir una tipología de mercados regulados por formas de integración como la reciprocidad, la redistribución y el intercambio.

Tomando en cuenta estas consideraciones se puede plantear la existencia de distintos tipos de mercado en función del grado de subjetivación alcanzado en las relaciones sociales y económico-contractuales que construyen los agentes que participan en él (cuadro 1) y que tendrían lugar en los barrios de origen informal.

\section{Cuadro 1. Posibles tipos de mercado informal de arrendamiento según el grado de subjetivación de la relación social y económica}

\begin{tabular}{|c|l|l|}
\hline $\begin{array}{c}\text { Grado de subjetivación de las } \\
\text { relaciones económicas y } \\
\text { contractuales }\end{array}$ & \multicolumn{2}{|c|}{ Grado de subjetivación de las relaciones sociales } \\
\hline Alto & Alto & Bajo \\
\hline Bajo & $\begin{array}{l}\text { Mercado informal } \\
\text { (redes) }\end{array}$ & $\begin{array}{l}\text { Mercado semi-informal } \\
\text { (anonimato) }\end{array}$ \\
\hline & $\begin{array}{l}\text { Mercado semi-formal } \\
\text { (redes) }\end{array}$ & $\begin{array}{l}\text { Mercado formal } \\
\text { (anonimato) }\end{array}$ \\
\hline
\end{tabular}


Un primer tipo de mercado corresponde a aquel donde las relaciones económicas y sociales son subjetivas. En este caso la relación de alquiler se establece entre familiares o amigos y esa relación social determina las características del compromiso contractual así como el precio. Se trataría del caso más típico de mercado informal en el cual lejos de prevalecer criterios mercantiles, operan criterios basados en una esfera doméstica que promueven mercados solidarios. En el otro extremo se encuentra el mercado donde la subjetivación de estas relaciones (económicas y sociales) es bajo y que corresponde a la figura clásica de mercado formal donde predomina el acceso en condiciones de anonimato y una valoración individual centrada en los lucros que dificulta el surgimiento de solidaridad alguna.

Las situaciones intermedias corresponden a situaciones en las cuales el grado de subjetivación de la relación social es alto, es decir pueden existir vínculos sociales de parentesco o amistad previos pero ellos no son tomados en cuenta para un trato diferenciado en la relación económica y contractual (razón por la cual se considera semi-formal) o en el caso contrario que exista una relación inicial anónima pero que en términos económicos opera de forma subjetivada (mercado semi-informal). Este último caso se ejemplifica en situaciones en las cuales el precio y/ o el acceso a una vivienda acontece a través de terceros quienes actúan como vínculo entre arrendatarios y arrendadores permitiendo a personas externas a la red ingresar a ella y pasar por un periodo de prueba en la cual surgen o se tejen nuevas relaciones sociales.

La tipología esquematizada es una propuesta que surge considerando que los barrios de origen informal presentan diferencias entre sí en materia de localización a nivel metropolitano, en relación al grado de consolidación y las mudanzas demográficas que operan a lo largo de su existencia. Cuando el tamaño de la población es pequeño se favorece la operación de las redes de relaciones y la subjetivación de las relaciones económicas que garantizan el funcionamiento del mercado. Sin embargo, al aumentar la población, especialmente de arrendatarios, se hacen más difíciles los mecanismos de control social dispuestos por las comunidades a partir de costumbres, normas y convenciones introduciéndose un mayor anonimato que puede promover formas de mercado cada vez más autorregulado. Debe agregarse que estos tipos pueden presentarse en la relación establecida por los agentes y cambiar con el tiempo, así pues una relación que inicia formalmente deriva con el tiempo en una informal a medida que se consolida la subjetivación social y económica.

Un último aspecto referido a la propuesta de tipologías de mercado se refiere al hecho de que los mercados informal, semi-formal y semi-informal permiten pensar también en diferentes escenarios donde opera la reciprocidad y de cómo la cooperación y coordinación entre los agentes es alcanzada en estos mercados. Desde este punto de vista se puede plantear entonces que un mercado solidario deberá combinar en sus justas proporciones la subjetividad y objetividad presente en las relaciones sociales y económicas para evitar no sólo una mercantilización del derecho a la vivien- 
da digna sino del otro lado a una situación en la cual si bien existe cierto tipo de reciprocidad, lo hace a expensas de mantener situaciones de dependencia, jerarquías basadas en la relación que se tiene con el jefe de familia o con aquel que funge como arrendador y/o dueño de la vivienda.

En relación al objetivo que impulsa a las familias de bajos ingresos a la subjetivación, personalización o informalización de la relación económica y contractual subyacen las expectativas por garantizar su reproducción biológica y social. Esta consideración expuesta por autores como José Luís Coraggio señala que la reproducción ampliada de la vida está asociada a una redefinición permanente de lo que dichas unidades consideran sus necesidades básicas. Estas no son estáticas y no existe un nivel básico dado porque las mejoras en la calidad de vida no presentan límites intrínsecos. Esas definiciones están determinadas por valores que son fruto de una construcción social divulgadas a través de la publicidad mercantil así como de diferentes movimientos culturales de la sociedad moderna, los cuales son interiorizados por esta población (Coraggio, 1998, 2005).

Lo anterior nos permite entonces pensar que la subjetivación de la relación económica y contractual lejos de ser una herramienta a la cual se apela por pura necesidad, en la medida en que se carece de la posibilidad de elección residencial va más allá y constituye una estrategia por la cual las familias intentan capturar las ventajas de localización que adquieren los barrios de origen informal más antiguos y consolidados. La cuestión que surge entonces es la de si esa estrategia permitirá también bloquear la tendencia a la gentrificación que se cierne como una de las principales amenazas para los barrios mejor localizados y que implica ante todo una alianza entre arrendatarios y propietarios por la defensa de sus territorios. 


\section{Notas}

1 Becario del Programa Estudantes-Convênio Pós-graduação ofrecido por la Coordenação de Aperfeiçoamento de Pessoal de Nível Superior PEC-PG CAPES Brasil. El siguiente trabajo presenta parte de los resultados presentados por el autor en su trabajo de tesis doctoral para optar por el título de Doctor en Planeación Urbana y Regional en el Instituto de Pesquisa e Planejamento Urbano e Regional da Universidade Federal do Rio de Janeiro IPPUR-UFRJ.

${ }^{2}$ La estratificación es un procedimiento utilizado por la Alcaldía Mayor de Bogotá para la aplicación de un esquema tarifario en materia de servicios públicos. Establece seis estratos de acuerdo a las características del entorno de la vivienda. Aquellos hogares que viven en barrios de estratos uno, dos y tres son subsidiados por las contribuciones realizadas por los hogares que viven en estratos cinco y seis, así como de quienes poseen predios destinados a fines distintos al residencial, mientras que las familias que residen en barrios de estrato cuatro pagan la tarifa plena (DAPD, 2004).

${ }^{3}$ Una consecuencia de la precaria definición de derechos de propiedad en este mercado corresponde a la inseguridad en la tenencia de la vivienda, entendida como la situación de desalojo forzado sea por parte del Estado o de un tercero que cuestiona dicha tenencia. Según cálculos presentados por la relatora para Naciones Unidas entre 1998 y 2008 cerca de 18 millones de personas en el mundo habían sido objeto de desalojos forzados, situación que constituía una crisis mundial en la inseguridad de la tenencia de la vivienda (ONU, 2012).

${ }^{4}$ Esta propuesta difiere de la utilizada por Pedro Abramo (2009) que se basa en la relación que tiene el objeto intercambiado (vivienda en alquiler) con la normatividad oficial. Según este autor cuando no opera regulación alguna de tipo oficial el mercado se considera informal. Si bien es importante reconocer la presencia total o parcial de un orden jurídico oficial se propone desplazar el foco de análisis del campo jurídico para las relaciones (sociales y económicas) existentes entre quienes intercambian. Al igual que menciona Abramo, esta propuesta no va en la dirección de esencializar lo informal sino de tomarlo en su forma descriptiva y pre-analítica, en este caso para la elaboración de la tipología de mercados. 


\section{Bibliografía}

Abramo, P. (2009a). "O mercado informal de solo em favelas e a mobilidade residencial dos pobres nas grandes cidades: um marco metodológico”. En: P. Abramo (Org.). Favela e mercado informal: a nova porta de entrada dos pobres nas cidades brasileiras,ANTAC, Porto Alegre, p. 14-47.

Ídem (2009b). “A cidade informal COM-FUSA: mercado informal em favelas e a produção da estrutura urbana nas grandes metrópoles latino-americanas”. En: P. Abramo, (Org.). Favela e mercado informal: a nova porta de entrada dos pobres nas cidades brasileiras, ANTAC, Porto Alegre, p.49-79.

Bassols, M. (1990). “La Marginalidad urbana: una teoría olvidada”. Revista Polis. Anuario de Sociología, n. 90, 181-198. Recuperado de http:// www.juridicas.unam.mx/publica/librev/rev/polis/cont/19901/pr/pr13.pdf

Castells, M. (1974). La cuestión urbana, Siglo XXI, Madrid.

Centro de estudios de la construcción y el desarrollo urbano y regional CENAC. (2007). Caracterización del mercado de arrendamiento urbano para el segmento de la población de bajos ingresos. Recuperado de http:// www.cenac.org.co/index.shtml?apc=I1----\&x=20152655

Ídem, (2012). Perfil del hogar arrendatario en Bogotá y estimación del valor del mercado. Recuperado de http://www.cenac.org.co/ index.shtml?apc=I1----\&x=20157819

Centro de Estudios de Opinión. C. E. O. (2002) Escuela Francesa de Sociología urbana: Compilación. Revista La Sociología en sus escenarios, no 6. Recuperado de http://aprendeenlinea.udea.edu.co/revistas/index.php/ceo/ article/view/1602

Coraggio, J. L. (1998). Economia popular. La perspectiva urbana, Abya Yala, Quito.

Ídem (2005). “Economia do trabalho”. En: C. Campolina \& M. Borges (Orgs.). Economia e Território, Editora UFMG, Belo Horizonte, p. 103129.

Cortes, F. (2002). Consideraciones sobre la marginalidad, marginación, pobreza y desigualdad en la distribución del ingreso. Papeles de población, Vol. 8, n. 31, 9-24. Recuperado de http://www.redalyc.org/ articulo.oa?id=11203101

De Soto, H. (2001). O mistério do capital, Editora Record, Rio de Janeiro.

Departamento Administrativo De Planeación Distrital DAPD. (2004). La equidad en las tarifas de servicios públicos y su impacto en la calidad de vida de los hogares de Bogotá, Bogotá. 
Germani, G. (1969). Sociología de la modernización, Ed. Paidós, Buenos Aires.

Jaramillo, S. (1980). Producción de vivienda y capitalismo dependiente: el caso de Bogotá, CEDE - UNIANDES, Bogotá:

Ídem (1993). El desenvolvimiento de la discusión sobre la urbanización latinoamericana. En: S. Jaramillo \& L.M. Cuervo. Urbanización latinoamericana: nuevas perspectivas, Escala, Bogotá.p. 9-41.

Ídem (1999). El papel del mercado del suelo en la configuración de algunos rasgos socioespaciales de las ciudades latinoamericanas. Revista Territorios, n. 2, p.107-129.

Lefebvre, H. (1972). La revolución urbana, Alianza Editorial, Madrid.

Ídem (1973). El derecho a la ciudad, Ediciones Península, Barcelona.

Organización de las Naciones Unidas ONU. Resolución A/HRC/22/46 de 24 de dezembro de 2012. Recuperado de http://direitoamoradia.org/wpcontent/uploads/2013/02/A.HRC_.22.46_sp.pdf

Parias, A. (2010). Mercado informal y estrategias residenciales de los sectores populares. En: S. Jaramillo (ed.). Bogotá en el cambio de siglo: promesas y realidade, OLACCHI, Quito, p. 293-336.

Parias, A \& Abramo, P. (2009). Mercado informal de suelo en los barrios populares de Bogotá: claves para entender el crecimiento de la metrópolis. En: P. Abramo (Org.). Favela e mercado informal: a nova porta de entrada dos pobres nas cidades brasileiras, ANTAC, Porto Alegre, p. 305-336.

Park, R. (1999). Ecología Humana. En: R. Park. La Ciudad y otros ensayos de ecología humana, Ediciones del Serbal, Barcelona, p. 127-140.

Ídem (1998). La ciudad: sugerencias para la investigación de la conducta humana en un ambiente urbano. Revista Colombiana de Educación, n. 3637, p.95-138. Recuperado de http://www.pedagogica.edu.co/storage/rce/ articulos/rce36-37_08tex.pdf

Polanyi, K. (2013a). El lugar de las economías en las sociedades. En: K. Polanyi. Textos escogidos. 1ra Edição. Los Polvorines, Universidad Nacional General Sarmiento, Buenos Aires, p. 83-86.

Ídem, (2013b). La economía como proceso instituido En: K. Polanyi. Textos escogidos. 1ra Ediçã, Los Polvorines, Universidad Nacional General Sarmiento, Buenos Aires, p. 87-112.

Schteingart, M. (2000). La investigación urbana en América Latina. Papeles de población, n.23, p. 9-27. Recuperado de http://www.redalyc.org/ articulo.oa?id=11202302 
Simmel, G. (1988). La metrópolis y la vida mental. En: M. Bassols, R. Donoso, A. Massolo, A. Méndez (Comps.), Antología de Sociología urbana, Universidad Nacional Autónoma de México, México, p. 47-61.

Stonequist, E. (1935). The problem of marginal man. American Journal of Sociology, Chicago, Vol. XLI, n. 1, p.1-12. Recuperado de http:// www.jstor.org/discover/10.2307/2768176? sid =21105621556 083\&uid=3737664\&uid=70\&uid=2129\&uid $=2$ \&uid $=4$

Topalov, C. (1979),La urbanización capitalista. Algunos elementos para su análisis, Edicol, México.

Torres, J. (2012)Estudio sobre el mercado de arrendamiento de vivienda en Colombia. Banco Interamericano de Desarrollo BID. Recuperado de http:/ /www.cenac.org.co/

Valencia, M. (2005). Escuelas y paradigmas de la ciudad moderna. Breve recorrido por los principales discurso del siglo $X X$, Centro de Estudios Arquitectónicos y del Paisaje, Facultad de Arquitectura, Urbanismo y Paisaje. Universidad Central de Chile, Santiago de Chile (Documento 2. Proyecto de Investigación CEAUP 2004-2005). Recuperado de http:// www.ucentral.cl/fid/investigacion_marco_valencia.html

Wirth, L. (1973). O urbanismo como modo de vida. En: G. Velho. O fenômeno urbano, Zahar Editores, Rio de Janeiro, p. 90-113.

Recibido: 30.10.16

Aceptado: 01.12.16 